\title{
Persistently high-level polyomavirus BK replication in the absence of renal function abnormalities in a kidney transplant recipient
}

\author{
Antonio Curtoni, ${ }^{1}$ Cristina Costa, ${ }^{1}$ Maria Messina, ${ }^{2}$ Francesca Sidoti, ${ }^{1}$ Andrea Piceghello, ${ }^{1}$ \\ Gabriele Bianco, ${ }^{1}$ Luigi Biancone, ${ }^{2}$ Giuseppe Paolo Segoloni, ${ }^{2}$ Rossana Cavallo' \\ ${ }^{1}$ Microbiology and Virology Unit, and ${ }^{2}$ Renal Transplantation Unit, Città della Salute e della Scienza \\ University Hospital, Turin, Italy
}

\section{Summary}

Polyomavirus-associated nephropathy is an important cause of allograft dysfunction and graft loss after kidney transplantation. Even if histological evaluation is the gold standard for graft study and diagnosis of polyomavirus-associated nephropathy, K-DIGO guidelines suggest performing an indication biopsy in selected patient's clinical conditions or laboratory parameters. The practice of protocol biopsy is still controversial. We report the management of a case of presumptive polyomavirus-associated nephropathy in a 53-year-old kidney transplant recipient affected by type 1 hyperoxaluria with persistent high levels of viruria and sustained levels of polyomavirus BK viremia. The presence of a presumptive polyomavirus-associated nephropathy, even if never confirmed by biopsy, never compromised his clinical condition and allograft function. As a result of an immunosuppression-sparing policy and use of mTOR inhibitor, the polyomavirus BK viremia was successfully controlled with an observation time $>5$ years. The deci-

Correspondence: Cristina Costa, Microbiology and Virology Unit, University Hospital Città della Salute e della Scienza di Torino, Corso Bramante 88, 10126 Turin, Italy.

Tel.: +39.11.6335953 - Fax: +39.11.6335194

E-mail: cristina.costa@unito.it

Key words: polyomavirus BK; kidney transplantation; polyomavirus-associated nephropathy; viral replication; real-time PCR.

Contributions: AC, FS, AP, GB, specimen collection and processing, data analysis;CC, study coordination, manuscript preparation; MM, LB, GPS, collection of clinical data, data analysis; RC, study coordination and supervision.

Conflict of interest: the authors declare no potential conflict of interest.

Received for publication: 26 May 2016.

Revision received: 19 July 2016.

Accepted for publication: 26 July 2016.

(C) Copyright A. Curtoni et al., 2016

Licensee PAGEPress, Italy

Microbiologia Medica 2016; 31:6031

doi:10.4081/mm.2016.6031

This article is distributed under the terms of the Creative Commons Attribution Noncommercial License (by-nc 4.0) which permits any noncommercial use, distribution, and reproduction in any medium, provided the original author(s) and source are credited. sion to perform or not a graft biopsy was the main question in the management of this case. We opted for a non-invasive approach because of the high risk of biopsy with macrohematuria on earlier biopsy in a dual kidney transplant and patient's unwillingness for the procedure. The replication level of polyomavirus BK was significantly reduced by the decrease of immunosuppression on the basis of a close nucleic acid testing monitoring. The strategy we adopted could be considered in cases when renal biopsy is contraindicated or considered to be high risk.

\section{Introduction}

Uncontrolled polyomavirus BK (BKV) replication is the main pathogenic factor responsible for polyomavirus-associated nephropathy (PVAN). The emergence of PVAN has been repeatedly associated with the introduction of new potent immunosuppressive regimens $(2,14)$. PVAN may develop in 1 up to 10\% of kidney transplant (KT) recipients, with a peak of incidence in the first year post-transplantation. Progression of PVAN leads to the allograft loss in more than $50 \%$ of the cases, with higher rates in the presence of late diagnosis or treatment failure (2).

Diagnosis and treatment can be challenging, as PVAN presentation is usually asymptomatic and may mimic rejection with a gradual rise of serum creatinine levels (2). Moreover, at renal agobiopsy examination, PVAN is often morphologically indistinguishable from cell-mediated acute rejection (12). To date, diagnostic tools for BKV-PVAN include: urinary citology for decoy cells identification, electron microscopy for viral aggregate detection in urine, nucleic acid testing (NAT) on serum and urine and the examination of renal graft biopsy. Biopsy examination is the diagnostic gold standard for the definitive diagnosis and grading of PVAN (12). We report the case of a 53-yearold KT recipient affected by type 1 hyperoxaluria with a persistently high level of BKV viremia suggestive of PVAN onset, successfully controlled by the decrease of immunosuppression on the basis of a close NAT monitoring on serum only.

\section{Case Report}

The patient was a 53-year-old male suffering from type 1 hyperoxaluria (3). Molecular genetics diagnosis revealed a compound heterozygosity on alanine glyoxylate aminotransferase gene, with a mutant allele associated with good response to vitamin B6 therapy. At the admission on the renal transplant waiting list, plasma oxalate con- 
centration was of $90 \mu \mathrm{mol} / \mathrm{L}$, increasing in spite of treatment with calcium-oxalate crystallization inhibitors.

In hemodialytic treatment since March 2009, he received a double KT in July 2009. Although HLA-matching was scarce, transplantation was performed considering the Turin Kidney Transplantation Center's allocation criteria for marginal donor and for the old-for-old program (15). Main patient's features are reported in Table 1.

Immunosuppressive protocol in the early post-operative period was based on a calcineurin inhibitor (CNI)-sparing regimen. Therefore, a sequential induction therapy with basiliximab $(20 \mathrm{mg}$ during surgery and $20 \mathrm{mg}$ on day 4) and anti-human thymocyte immunoglobulin (2 $\mathrm{mg} / \mathrm{kg} /$ day) was administered to minimize nephrotoxicity. Due to an allergic reaction, anti-human thymocyte immunoglobulin was withdrawn, followed by steroid and antihistamine administration. At +2 days post-KT, tacrolimus was introduced; at +9 days, serum creatinine was $1.46 \mathrm{mg} / \mathrm{dL}$. The maintenance immunosuppressive regimen at discharge included tacrolimus, mycophenolate mofetil (MMF, $2 \mathrm{~g} /$ day) and steroid with a target therapeutic range of tacrolimus of $10-13 \mathrm{ng} / \mathrm{mL}$ by antibody-conjugated magnetic immunoassay.

At +1 month post-KT, on the basis of clinical suspicion of acute rejection, in the presence of increasing serum creatinine and unstable tacrolimus levels, a steroid bolus was administered and ultrasoundguided fine needle biopsy was performed. During the procedure, bleeding complication with macrohematuria occurred, although with stable hemoglobin levels, not requiring transfusions. At histological examination, acute rejection was excluded and a pattern of CNI nephrotoxicity was reported.

Polyomavirus BK-DNA level in urine was $>5 \times 10^{6}$ copies $/ \mathrm{mL}$, with no evidence of viremia; immunohistochemistry with cross-reacting monoclonal antibodies against the large T-antigen of the Simian polyomavirus SV40 and NAT on renal biopsy were negative. To reduce the nephrotoxic effects of CNI, tacrolimus was tapered.

At +48 days, the patient presented stable renal function and was discharged with tacrolimus and creatinine levels of $8-10 \mathrm{ng} / \mathrm{mL}$ and 1.47 $\mathrm{mg} / \mathrm{dL}$, respectively.

In the following months, virological monitoring for BKV evidenced viruria levels persistently $>5 \times 10^{6}$ copies $/ \mathrm{mL}$ in the presence of viremia levels $<10^{4}$ copies/mL, thus indicating a high local replication rate with serum BKV and creatinine levels not suggestive of PVAN.

At +4 months post-KT, the therapeutic regimen was modified in order to reduce the net amount of immunosuppression: MMF was withdrawn; an extended-release formulation of tacrolimus was adopted with target levels of 5-7 ng/mL to be further reduced within 3-4 months; the mTOR inhibitor everolimus (range $5-8 \mathrm{ng} / \mathrm{mL}$ ) was introduced to exploit its anti-polyomavirus activity $(1,16)$.

In the following months up to +24 , BKV viremia increased and reached levels almost persistently $>10^{4}$ copies $/ \mathrm{mL}$ serum with peak values $>5 \times 10^{6}$; viruria was persistently $>5 \times 10^{6}$ copies $/ \mathrm{mL}$ throughout the whole period. Nevertheless, serum creatinine levels were stable with a mean value of $1.2 \mathrm{mg} / \mathrm{dL}$. In order to evaluate the polyomavirus BK-specific cellular immune response, an Enzyme-linked Immunospot
(Elispot) assay was performed at +15 and +17 months and showed the lack of virus-specific immune response. Despite BKV viremia levels persistently high, no biopsy was performed to investigate the presence of PVAN and the histological pattern, based on the clinical conditions with persistently good graft function, the previous bleeding episode and the reluctance of the patient towards the bioptic procedure (9).

Subsequently, tacrolimus and everolimus target levels were further reduced to $1.5 \mathrm{ng} / \mathrm{mL}$ and $4 \mathrm{ng} / \mathrm{mL}$, respectively, in 2-3 months. Steroid dose was tapered to $2.5 \mathrm{mg}$ every other day, too. BKV viremia reached the climax of $5 \times 10^{6}$ copies/mL at +16 months then gradually decreased to $\leq 10^{3}$ copies/mL starting from +24 months. The kinetics of BKV viremia and serum creatinine levels during the follow-up are plotted in Figure 1.

At the end of the follow-up which is now longer than five years, the patient's renal function is stable with serum creatinine level persistently $\leq 1.2 \mathrm{mg} / \mathrm{dL}$; polyomavirus BK viremia is monitored twice a year (last determination 200 copies $/ \mathrm{mL}$ ). Proteinuria was always in the non pathological range. Patient's clinical conditions are excellent, he is a full-time worker and a sportsman.

Many studies have focused on PVAN over years, but numerous questions are still unanswered. In this case KT-related risk factors, BKV viremia onset-time and sustained viral load should suggest an underlying pattern of severe PVAN but the evolution of allograft function during therapeutic management and long-term follow-up is in contrast

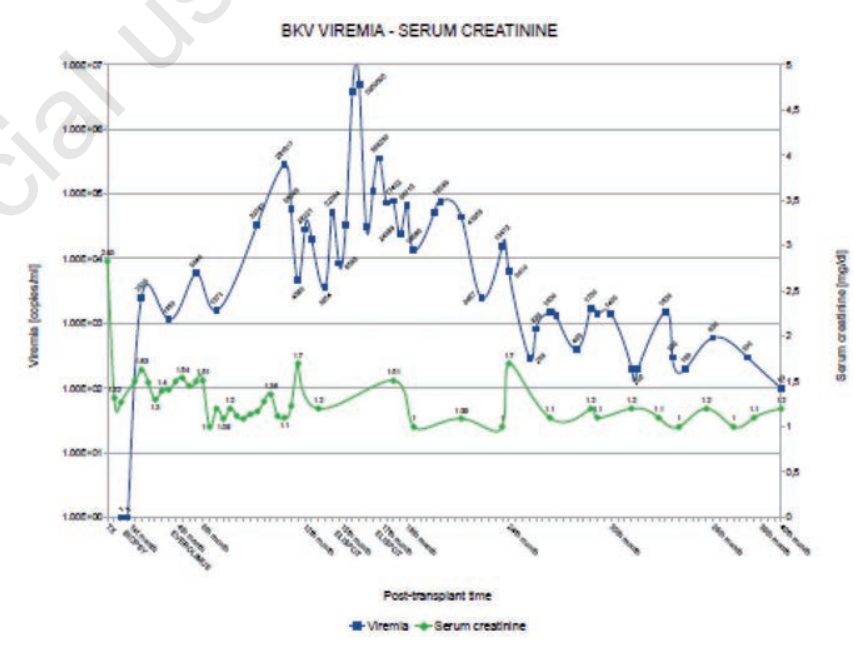

Figure 1. Polyomavirus BK viral load and serum creatinine. In the figure are reported BKV viremia (blue line) and serum creatinine (green line) levels in function of time during the 5-year follow-up post-KT. For each immunosuppressive drug target levels are indicated. MMF, mycophenolate mofetil; TAC, tacrolimus; EVR, everolimus; KT, kidney transplantation.

Table 1. Main clinical features of the patient. Patient features

Age; gender

52; Male

Underlyng disease

Type 1 hyperoxaluria; mutations: c.33dupC (exon) 1), c.508G >A (exon 4)

Transplant type

Double deceased-donor kidney transplant

HLA

Donor: A 2-31(19) B 44(12) 60(40) DR 4-4

Recipient: A 30(19) 32(19) B 14-27 DR 4-13(6)

CMV matching

$\mathrm{D}-/ \mathrm{R}+$ 
with this hypothesis. Non invasive diagnostic methods have low positive predicting value, lack of standardization and don't provide prognostic information, however in clinical practice a presumptive diagnosis of PVAN mandatorily require a therapeutic intervention (13).

\section{Discussion and Conclusions}

The PVAN onset can be supposed (possible PVAN) in the presence of high levels of BKV replication in urine by the identification of decoy cells or viral particles at the electron microscopy or in the presence of viral loads $>10^{7}$ copies/mL urine $(2,9)$.

A presumptive diagnosis of PVAN can be made by detection of BKVDNA $>10^{4}$ copies/mL in serum for $>3$ weeks. Identification of decoy cells and NAT on urine have a high negative predictive value, while NAT on serum has a high positive predictive value $(2,9)$.

This patient presented clinical features associated with a high risk to develop PVAN considering the following donor and recipient determinants: HLA-mismatching, deceased donation, male recipient, induction therapy with anti-human thymocyte immunoglobulin, and maintenance with triple immunosuppression regimen (10). Elispot assay revealed the lack of polyomavirus BK-specific T-cell response, thus suggesting the failure of patient's immune-surveillance $(5,6)$. According to PVAN definition criteria, in this patient, a presumptive diagnosis of PVAN could be made, as evidenced by a prolonged ( $>3$ weeks) BKV viremia $>10^{4}$ copies/mL $(2,9)$.

The decision to perform or not a graft biopsy was the main question in the management of this case. As a matter of fact, no biopsy was performed to investigate the occurrence of PVAN. Even if histological evaluation is the gold standard for graft study and diagnosis of PVAN, KDIG0 guidelines suggest the execution of renal graft biopsy only in some circumstances and further studies should be performed to properly define the benefits of protocol biopsy particularly in terms of therapeutical interventions (11). In this patient, this choice was made on the basis of stable and excellent graft function, thus avoiding an invasive procedure with a potential risk of a repeated bleeding complication, a main troublesome anxiety in the patient. Moreover the multifocal nature of PVAN could also be a point in favour of the decision in this case (8).

Although no definitive diagnosis could be done in the absence of histological evaluation, in this patient, therapeutic intervention was successfully based on BKV viremia, whereas no decisional role was played by BKV viruria (4).

The modulation of the immunosuppressive regimen appeared to be the more suitable approach, as it lead to the inhibition of viral replication and PVAN progression (7). Early identification of BKV replication by a close monitoring and a pre-emptive reduction of immunosuppression, possibly using a mTOR inhibitor drug, tapering $\mathrm{CNI}$ as much as possible, represent an adequate strategy to manage viral reactivation and prevent PVAN development. Even if we were aware of the elevated molecular monitoring cost and of the fact that the histological picture is the gold standard for defining the entity and the type of renal damage caused by $\mathrm{BKV}$, the strategy we adopted can be retrospectively defined as a successful one in our patient and it avoided the bioptic procedure when BKV viremia was at high levels. In cases when renal biopsy is contraindicated or highly riskful, provided that serum creatinine does not significantly increase, this strategy could be considered a valuable option.

\section{References}

1. Benavides CA, Pollard VB, Mauiyyedi S, et al. BK virus-associated nephropathy in sirolimus-treated renal transplant patients: incidence, course, and clinical outcomes. Transplantation 2007;84:83-8.

2. Bohl DL, Brennan DC. BK Virus nephropathy and kidney transplantation. Clin J Am Soc Nephrol 2007;2:S36-46.

3. Cochat P, Hulton SA, Acquaviva C, et al. Primary hyperoxaluria Type 1: indications for screening and guidance for diagnosis and treatment. Nephrol Dial Transplant 2012;27:1729-36.

4. Costa C, Bergallo M, Astegiano S, et al. Monitoring of BK virus replication in the first year following renal transplantation. Nephrol Dial Transplant 2008;23:3333-6.

5. Costa C, Mantovani S, Piceghello A, et al. Evaluation of polyomavirus BK cellular immune response by an Elispot assay and relation to viral replication in kidney transplant recipients. New Microbiol 2014;37:219-23.

6. Costa C, Saldan A, Cavallo R. Evaluation of virus-specific cellular immune response in transplant patients. World J Virol 2012;1:150-3.

7. Hardinger KL, Koch MJ, Bohl DJ, et al. BK-virus and the impact of pre-emptive immunosuppression reduction: 5-year results. Am J Transplant 2010;10:407-15.

8. Hirsch HH. BK virus: opportunity makes a pathogen. Clin Infect Dis 2005;41:354-60.

9. Hirsch HH, Brennan DC, Drachenberg CB, et al. Polyomavirusassociated nephropathy in renal transplantation: interdisciplinary analyses and recommendations. Transplantation 2005;79:1277-86.

10. Hirsch HH, Randhawa P. AST infectious diseases community of practice: BK polyomavirus in solid organ transplantation. Am J Transplant 2013;13:179-88.

11. Kidney Disease: Improving Global Outcomes (KDIG0) Transplant Work Group. KDIGO clinical practice guideline for the care of kidney transplant recipients. Am J Transplant 2009;9:S1-155.

12. Meehan SM, Kadambi PV, Manaligod JR, et al. Polyoma virus infection of renal allografts: relationships of the distribution of viral infection, tubulointerstitial inflammation, and fibrosis suggesting viral interstitial nephritis in untreated disease. Hum Pathol 2005;36:1256-64.

13. Nickeleit V, Singh HK. Polyomaviruses and disease: is there more to know than viremia and viruria? Curr Opin Organ Transplant 2015;20:348-58.

14. Ramos E, Drachenberg CB, Wali R, Hirsch HH. The decade of polyomavirus BK-associated nephropathy: state of affairs. Transplantation 2009;87:621-30.

15. Segoloni GP, Messina M, Squiccimarro G, et al. Preferential allocation of marginal kidney allografts to elderly recipients combined with modified immunosuppression gives good results. Transplantation 2005;80:953-8.

16. Wali RK, Drachenberg C, Hirsch HH, et al. BK virus-associated nephropathy in renal allograft recipients: rescue therapy by sirolimusbased immunosuppression. Transplantation 2004;78: 1069-73. 